Travail immatériel et immesurable?

\title{
Le travail de l'orientation. Notes sur des textes d'Alain Badiou (et de quelques autres)
}

\section{Andrea Cavazzini}

\section{OpenEdition}

\section{Journals}

Édition électronique

URL : http://journals.openedition.org/grm/874

DOI : $10.4000 / g r m .874$

ISSN : 1775-3902

Éditeur

Groupe de Recherches Matérialistes

\section{Référence électronique}

Andrea Cavazzini, « Le travail de l'orientation. Notes sur des textes d'Alain Badiou (et de quelques autres) », Cahiers du GRM [En ligne], 10 | 2016, mis en ligne le 18 décembre 2016, consulté le 01 mai 2019. URL : http://journals.openedition.org/grm/874 ; DOI : 10.4000/grm.874

Ce document a été généré automatiquement le 1 mai 2019

(c) GRM - Association 


\title{
Le travail de l'orientation. Notes sur des textes d'Alain Badiou (et de quelques autres)
}

\author{
Andrea Cavazzini
}

\section{«Orientation »}

1 Il semble que nous sommes entrés dans une conjoncture dont les perspectives politiques sont opaques, et que les quelques espoirs qui avaient pu surgir timidement, en Europe et ailleurs, dans les années précédentes, concernant un changement dans les rapports de force et dans l'organisation de la société, ont été engloutis par la stabilisation des pouvoirs en place.

2 L'horizon immédiat est de plus en plus saturé par les images envahissantes de la guerre et de la terreur, l'initiative politique semble être monopolisée par les armées, les États et les polices, les fausses alternatives et les mots d'ordre piégés occupent l'espace laissé par le recul des perspectives réelles de transformation. «Pas une voix, hormis la voix de ceux qui règnent,

Et sur tous les marchés l'exploitation proclame : c'est maintenant que je commence » (Brecht, Eloge de la dialectique).

3 Devant un tel état des choses, est-il possible d'indiquer malgré tout des points de repères - et donc de s'orienter?

4 Dans une intervention politique récente, Alain Badiou affirme que «le temps présent, dans un pays comme la France est, depuis trente ans, un temps désorienté $»^{1}$ :

Quel peut être aujourd'hui le principe et le nom d'une orientation véritable? Je propose en tout cas de l'appeler, par fidélité à l'histoire des politiques d'émancipation, l'hypothèse communiste $e^{2}$.

5 Le mot d'orientation choisi pour caractériser l'hypothèse communiste n'est évidemment pas anodin, aussi il y aura peut-être quelques intérêts à le déplier. 
6 C'est probablement avec Kant que le terme d'«orientation » accède à une dignité proprement philosophique en finissant par indiquer l'acte le plus important et décisif de la pensée. Kant caractérise l'orientation dans la pensée par une analogie avec l'orientation spatiale consistant à «trouver à partir d'une région céleste donnée (...) les autres régions et surtout l'orient $»$ :

A cet effet il m'est indispensable d'éprouver par rapport à moi-même le sentiment [ Gefühl] d'une différence ; je veux dire celle de la droite et de la gauche. Je me sers du terme de sentiment, car vus du dehors ces deux côtés ne présentent dans l'intuition aucune différence notable (...). Par conséquent, compte tenu de toutes les données objectives, je ne m'oriente géographiquement qu'au moyen seulement d'un principe de différenciation subjectif ${ }^{3}$.

7 Ce principe de différenciation subjectif, que Kant rattache à la sphère du Gefühl - le sentiment, le ressenti, l'affect - suffit pour déterminer une situation à l'égard de laquelle aucun point de repère objectif n'est donné. C'est la situation qu'on retrouve dans la sphère de la raison lorsqu'elle "cherche à s'élever par delà toutes les bornes de l'expérience et ne trouve absolument rien comme objet d'une intuition » :

La raison n'est alors plus en état de soumettre ses jugements à une maxime positive d'après les principes objectifs de la connaissance ; elle ne peut que se fonder sur un principe subjectif de différenciation. Ce principe subjectif (...) n'est rien d'autre que le sentiment du besoin inhérent à la raison ${ }^{4}$.

8 Il y a donc quelque chose comme un sentiment qui est propre à la raison, et qui renvoie au besoin que celle-ci éprouve de déterminer des situations par delà les connaissances objectives disponibles.

9 Or cette disjonction d'avec les connaissances peut être motivée provisoirement par une insuffisance de celles-ci. Mais la thèse de Kant est plus radicale. Elle porte sur ce qui, dans la pensée, excède tout savoir et qui correspond néanmoins à la fonction décisive de déterminer une situation.

Il est donc question, dans l'orientation, d'un usage de la pensée par delà le savoir et qui implique la subjectivité comme telle. Or de quelle forme d'implication s'agit-il? La subjectivité qui est ici évoquée est caractérisée d'abord à travers le besoin - c'est donc le sentiment d'un manque qui qualifie initialement cette subjectivité. Le sentiment subjectif vient du fait que la raison a besoin de quelque chose. De quoi? «De se tracer une voie appropriée au but total de sa destination $»^{5}$. Il est donc question d'une subjectivité définie à la fois par un manque - absence de repères objectifs, sentiment d'un besoin - et par une aspiration à la totalité. Telle est donc la structure de l'orientation.

11 Alexander Kluge a repris la problématique kantienne du point de vue des voies paradoxales qu'empruntent les forces cachées qui soutiennent l'agir et la survie des hommes dans l'histoire :

Nous devons nous orienter. Pour cela nous disposons d'une série de propriétés plus ou moins utiles et fiables: l'inertie, l'accoutumance, le blindage, le dévouement, l'abnégation, l'infaillibilité (d'un pickpocket), l'insatiabilité de la raison, le bonheur en récompense de la vertu ${ }^{6}$.

12 Tout comme le kantien «besoin de la raison» (qui est d'ailleurs évoqué assez ouvertement), les propriétés que Kluge énumère constituent le «fond » non-thématique et préconscient de nos déterminations rationnelles, tant pratiques que théorétiques, que résume le mot de « sentiment » (Gefühl) :

Ce dont les hommes ont besoin au cours de leurs vies, c'est d'orientation (...).

L'orientation subjective - savoir à quoi me fier, ce que je dois craindre, ce qui sous 
tend les actes volontaires - donne ce courant de fond, que le temps n'altère en rien ${ }^{7}$ capitalistes et néo-impériales, Alain Badiou pose une "grande et centrale question politique » :

Où se joue (...) une volonté effective non seulement de freiner, d'enrayer, le processus en cours, mais de l'interdire, c'est-à-dire d'ouvrir à un processus entièrement différent ? ${ }^{12}$ mouvement communiste à construire une telle volonté et à ouvrir de tels processus de 
freinage et d'invention. C'est notre expérience depuis de décennies : tous les mouvements de contestation et de protestation qui se sont succédés depuis la fin des années 1990 ont été incapables, non seulement d'imposer un rapport de forces favorables à leurs revendications, mais aussi de garder leur consistance face à des échecs et à des impasses.

Mouvements anti-globalisation, mobilisations contre les guerres impériales (au Kosovo, en Afghanistan, en Iraq...), mouvements suscités par la crise économique de 2007, par la crise grecque - et bien entendu les révoltes dans le monde arabe, la tentative grecque... Il est difficile de nier que toutes ces mobilisations, expériences et tentatives semblent avoir été incapables de laisser suffisamment de traces, de modifier les rapports de forces, mais aussi de s'inscrire dans les subjectivités, d'une manière assez profonde. Le Gefühl qui a été théorisé en tant que base affective des mobilisations issues de la crise financières l'indignation - est lui-même un affect par définition lié à des réactions immédiates et donc difficile à inscrire dans une durée.

21 La question posée par Badiou semble rendre équivalents le problème de la formation d'une « volonté effective » et celui de la capacité à exercer des effets réels sur les rapports et les puissances du capitalisme mondialisé. C'est que nulle volonté ne saurait être " effective " sans produire un changement réel dans les rapports de forces, et que la construction durable d'une telle volonté est déjà un acte relevant de l'effectivité politique.

L'effectivité de la volonté est au cœur de la pensée dialectique de la guerre développée par Clausewitz. Oskar Negt a rappelé que selon celui-ci «la guerre est un "acte de violence destiné à forcer l'adversaire à accomplir notre volonté" $»^{13}$. La neutralisation de l'ennemi consiste à le priver de son autodétermination, à faire qu'il se soumette à une loi extérieure qu'il ne s'est pas donnée. Se soumettre à la volonté d'autrui signifie surtout renoncer à persévérer dans son être propre, renoncer en quelque sorte à «tenir ». Ainsi, l'effectivité de la volonté renvoie encore une fois à quelque chose qui est de l'ordre de la consistance et de la persistance ; loin de se réduire à un vœu ou à un souhait immatériels, la volonté est une force efficace, mais uniquement à condition d'indiquer une capacité à « tenir » des points et des positions.

23 C'est donc au problème des dispositifs subjectifs qui organisent cette capacité que nous sommes renvoyés. Alexander Kluge a proposé une méditation sur les thèmes clausewitziens en rattachant ces dispositifs à des compositions de sentiments. Une prose de l'écrivain allemand présente un spécialiste des stratégies de la Guerre Emotionnelle, William Friedrichsen, constatant que, dans les guerres, « on prend des décisions en vertu de facteurs qui, en langage non scientifique, s'appellent des sentiments » :

Les sentiments étaient vraiment aux commandes partout. Sont identifiables : peur, amour, espérance, nostalgie, deuil, dévouement, ainsi que tout le complexe de l' inertie des sentiments ${ }^{14}$.

24 Les sentiments articulent l'action et l'invariance, la transformation et la conservation, en rattachant la capacité d'agir à l'entretien d'une réserve virtuelle de dispositions :

Les sentiments se préservent des atteintes grâce à leur facteur d'inertie (...), mais (...

) ils font preuve de vigilance dès qu'à côté du mouvement inerte apparaît un élément quelconque, surprenant et inexpliqué, qui a jusque-là échappé à l'entendement ${ }^{15}$.

25 Ainsi, les sentiments permettent non seulement d'agir, mais de conserver le potentiel de l'action et de renouveler la disposition à agir : 
Chacun de façon différente, ils renfermaient un faible élan collectif, chaque fois réprimé par un fort potentiel d'excitation qui se décharge rapidement sous la forme d'une salve complète pour revenir ensuite au repos et préparer un nouveau tir ${ }^{16}$. selon Friedrichsen, « d'ôter à ces nuées émotionnelles les excitations qui les déclenchent ou d'interrompre prématurément les processus de leur formation $\aleph^{17}$. Les stratégies développées par Friedrichsen pour priver l'ennemi de la force de ses propres sentiments rappellent l'expérience réelle de populations innombrables frappées par les bombardements, la guerre civile, l'effondrement de l'économie ou le pur et simple déclin graduel des conditions de vie :

Il est évident pensait Friedrichsen, que ce que j'aime ou protège constitue une partie essentielle de la force armée des sentiments. Comment priver l'adversaire de ce pouvoir essentiel qui est le sien? (...). Comment, demandait Friedrichsen, allonsnous faire pour bombarder l'espoir? Je réduis en miettes le temps requis par le travail de deuil. Je fais feu sur les pauses minuscules qui composent le facteur d'inertie et la rechute dans l'apaisement des émotions. Un être foncièrement inquiet est inapte à la vie et anéanti dans sa volonté18.

Mais ces êtres dont toute consistance a été annulée ne deviennent pas pour autant absolument dociles : au contraire, la perte de toute capacité de s'orienter les transforme en des machines de guerre implacables:

Quand je n'ai plus rien à perdre, alors j'ai perdu aussi mon angoisse (...). Si espoir deuil, inertie et tranquillité sont complètement liquidés, il ne reste alors à l'adversaire plus rien qui puisse capituler (...) ou qui s'accorde avec nous pour mettre fin à la guerre ${ }^{19}$.

\section{« Guerre »}

L'évocation de la guerre est omniprésente aujourd'hui. Il est très dangereux, et désorientant, de se laisser interpeller par cette évocation comme si elle définissait une situation absolument nouvelle et inédite. Comment s'étonner devant l'éclatement de violences ouvertes et de conflits militaires directs alors que l'état de guerre est la situation ordinaire des sociétés capitalistes, et que, après 1989, cette guerre est déclarée de manière unilatérale contre les classes laborieuses? C'est l'enjeu de la méditation sur Clausewitz proposée par Oskar Negt :

La simple absence d'un conflit militaire ne définit pas encore l'état pacifique d'une société (...). Il existe au sein de notre société un grand nombre d'actes de guerre qui se cachent sous l'auréole de la paix ${ }^{20}$.

Oskar Negt fait allusion aux conséquences du déchaînement de l'accumulation du profit capitaliste :

L'un des problèmes décisifs de notre société réside dans le fait qu'un nombre croissant de personnes vivent dans un état d'insatisfaction et d'angoisse existentielle (...). La croissance et l'excroissance des formes de violence - se déchargeant dans des actions apparemment dénuées de raison - se développent sur le sol fertile d'une société qui n'est pas en mesure de répartir ses vastes richesses sociales d'une manière appropriée ${ }^{21}$.

Cette incapacité à répartir les richesses ne concerne pas que la simple distribution des ressources; elle touche à l'usage ou à l'inutilité des existences, tels que les révèle le chômage entre autres phénomènes : 
Le chômage représente le pillage et l'expropriation des facultés et qualités que des personnes ont difficilement acquises à travers un processus de formation, impliquant la famille, l'école et la formation professionnelle (...) et qui courent alors le danger de péricliter ${ }^{22}$.

31 La production d'une vaste masse d'« hommes inutiles » est l'un des traits fondamentaux de la phase actuelle du monde capitaliste. Elle ne concerne pas que la gestion de la forcetravail, mais porte principalement sur la constitution subjective. La guerre permanente que représentent les processus contemporains de l'accumulation capitaliste fait partie des stratégies visant à déstructurer totalement la composition subjective de l'ennemi virtuel que la force-travail est toujours du point de vue du capital.

32 Ainsi, ces masses affectées par l'inutilité, la précarité et la peur deviennent incapables de faire "tenir » leur volonté face à celle de l'ennemi - dès lors, elles deviennent aussi incapables de retenir et de freiner la violence qui les traverse et dont elles deviennent des relais ou des échangeurs aveugles. Elles répercutent d'une manière tantôt aléatoire tantôt savamment orchestrée, l'état d'angoisse et l'exposition à la violence dont leurs affects sont constamment ébranlés. La brutalité, les humiliations, la peur, le gaspillage et la mutilation des existences qu'une société engendre en reproduisant ses privilèges et ses inégalités ne disparaissent pas ; les mensonges, l'abêtissement volontaire, la lâcheté et les trahisons dont elle a besoin pour dénier les horreurs dont elle se nourrit ne disparaissent pas non plus. Ils ne font que se déposer dans des latences immémoriales, d'où ils resurgissent sous la forme de fantasmes et de conduites parfois fort éloignés de leurs racines archi-historiques, dont ils traduisent néanmoins la violence cachée :

Ces histoires nous parlent d'aujourd'hui et d'événements qui remontent à douze mille ans (...). Et cependant l'être humain évolue si peu. Pour moi, la bibliothèque d'Alexandrie brûle encore de nos jours ${ }^{23}$.

33 L'inertie des sentiments n'est pas seulement une ressource; elle fixe et transmets des mutilations, engendre des spectres. Les réseaux invisible qui composent la toile de l'Histoire n'ont de cesse de faire ressurgir les blessures et les refoulements :

[Lors de la révolte des tisserands silésiens en 1844] dans les métropoles européennes, la révolte et sa répression sanglante faisaient l'objet de débats publics. Dans l'Eulengebirge, en revanche, la colère se transforma, lorsqu'elle s'étendit des tisserands et des participants directs de l'émeute aux témoins de l'événement et à la population qui n'y avait pas pris part. La colère et la fureur se muèrent en une attitude subversive, mais prudente. Si l'on voulait survivre, il importait de camoufler ses convictions séditieuses. Affecter un tel comportement a cependant des répercussions sur l'intériorité (...). Les salariés cherchant protection se sont rapprochés des propriétaires fonciers. Bien qu'affranchis officiellement en 1807, ils étaient malgré tout réduits au servage (...). Mon grand-père maternel (...) a vingt-six ans lorsqu'il assiste à l'écrasement de la révolte des tisserands. Il vient alors d'être démobilisé. S'il avait été du camp des rebelles, il aurait eu les qualités d'un meneur (...). Au lieu de cela, il s'engage en tant que volontaire dans la réserve de la Landwehr, laquelle réprime la révolution à Berlin en 1848 (...). Cette fidélité peut même se nourrir d'une agressivité détournée, d'une colère intérieure, de manière à intensifier la collaboration ${ }^{24}$.

Les migrations et les traductions des sentiments scandent les constitutions historiques des subjectivités. Elles sont les opérateurs d'une économie archi-historique qui fait que nul acte ne restera sans conséquences, bien que celles-ci puissent se manifester sous des formes indirectes et méconnaissables. C'est pourquoi lorsqu'Alain Badiou dit que «notre mal vient de plus loin que l'immigration, que l'Islam, que le Moyen-Orient dévasté, que l'Afrique soumise au pillage... Notre mal vient de l'échec historique du communisme $»^{25}$, il 
faut entendre ces mots autrement que comme une provocation. Car les conséquences subjectives d'un tel échec, articulées au déchaînement des esprits animaux capitalistes et impériaux, ne sont pas encore entièrement mesurables. Nous n'avons fait que commencer à reconnaître leurs effets.

\section{«Idées »} Ainsi, le problème se pose de savoir si l'hypothèse communiste est destinée à rester un objet intellectuel, une «idée » au sens d'un principe qui n'a de conséquences pratiques que pour ceux qui croient à la force des principes - les « intellectuels », justement. Mais la question porte aussi sur les affects qui définissent ces êtres que nous qualifions d'« intellectuels ». appartiennent au champ des pratiques traditionnellement qualifiées d'« intellectuelles ». D'ailleurs, la note de lecture, la recension, la critique, en tant que gestes transposant des significations d'un système initial de repères vers des champs différents, constituent peut-être des paradigmes de la pratique intellectuelle en tant que telle, dans la mesure où de tels gestes supposent ou appellent implicitement l'existence d'une "conversation", d'une « société » en tant que solidarité entre des êtres libres, dont l'échange des paroles et des actes réalisent un certain partage de l'activité et du sens.

Mais il y encore d'autres raisons. Entre 2006 et 2007, Alain Badiou a entamé, dans des ouvrages comme Le siècle ou De quoi Sarkozy est-il le nom ?26, une réappropriation critique de l'expérience du communisme ayant comme but explicite l'ouverture d'une nouvelle séquence dans l'existence de l'hypothèse communiste. Cette réappropriation a été à la fois le déclencheur et le symptôme d'une fissure dans le consensus idéologique (anticommuniste) des années 1980 et 1990, et a produit des effets incontestables dans le champ intellectuel : ces dix dernières années, le mouvement communiste du XXe siècle et ses différents aspects - le marxisme, la révolution, les luttes ouvrières... - sont redevenus des objets de pensée à part entière. L'Université et l'industrie culturelle ont enregistré ce phénomène, en le récupérant suivant leurs fins immanentes; il n'en reste pas moins que quelque chose s'est passé d'irréductible aux modes culturelles et à la reproduction institutionnelle, quelque chose qui était motivé par un désir authentique de comprendre et de penser par-delà les schémas dominants, de se réapproprier un passé récent et refoulé pour cerner les manques et les impasses du présent. d'envergure aient incarné depuis un bilan pratique des expériences du XXe siècle - un bilan qui ne peut être mené qu'en réinvestissant pratiquement les points dont l'éclipse du communisme a fait des tabous et des impensés : l'action politique des dominés, la maitrise des processus économiques, le rapport au pouvoir étatique... Une réappropriation de l'expérience communiste dans le champ politique impliquerait l'apparition de quelque chose de nouveau par rapport à ces points - une nouveauté susceptible de modifier les rapports de force actuels ou d'indiquer une voie possible pour leur modification.

Or c'est précisément de ces processus que nous constatons l'absence ou l'inefficacité. 
39 Kant parle d'un affect (Gefühl) qui est en même temps un «besoin de la raison ». Dire que la recherche d'une orientation, donc de l'expression d'une totalité, consiste en un besoin de la raison, implique que la raison est travaillée par des dynamiques affectives intrinsèques, mais aussi que les sentiments qui agissent dans cette recherche sont indissociables du déploiement d'une rationalité. Les fonctions rationnelles expriment un désir de totalité et une tendance vers la forme qui s'opposent à la dispersion et à la fragmentation de l'expérience. Dans ces fonctions, objets et dispositions que nous appelons «intellectuels», quelque chose se fait jour qui renvoie à l'image d'une expérience pleine, d'une activité libre, d'un monde consistant. Par là, ils jouent un rôle décisif dans la constitution d'une volonté politique effective :

Si on entend par « intellectuel » celui qui est capable de formaliser les principes à

l'égard d'une situation de masse, toute politique exige des intellectuels ${ }^{27}$.

Par « intellectuels » il faut entendre ici ceux qui parviennent à déterminer une situation suivant des principes - ce qui correspond au geste même de l'orientation. Déterminer une situation veut dire la penser du point de vue d'une totalité visée par la raison : par là, la situation contingente est inscrite dans la consistance d'une forme. Or les formes conceptuelles et discursives qui opèrent cette totalisation rationnelle n'actualisent leurs virtualités libératrices qu'en s'articulant avec des sphères affectives, des Gefühle, qui relèvent de strates plus profondes et cachées. Frédéric Lordon qualifie d'« intellectuel » tout individu (au sens spinozien d'une unité singulière de pouvoirs et de passions) dont la constitution singulière fait qu'il est « affectable » par des idées :

\footnotetext{
Il s'agit d'une affectabilité qu'on pourrait dire « intellectuelle » (...) qui a moins besoin des choses mêmes et peut se trouver activée par la simple "idée » des choses - en réalité par leur imagination, induite par des signes (et, comme toujours, accompagnée par des corrélats idéels) (...). Être disposé aux révoltes logiques, c'est être capable de convoquer en soi des images vives de choses absentes, et de les rappeler à soi par liaison avec d'autres images vues, mais pauvres, les images des signes, des mots écrits ${ }^{28}$.
}

Une "révolte logique » est une entité aussi paradoxale que le «besoin de la raison »: dans l'un comme dans l'autre cas, il s'agit d'articuler des affects à des formations « idéelles " pour faire en sorte que de cette articulation surgisse la capacité à traiter les « choses absentes». Or cette articulation est rendue possible par les trajets singuliers des affects :

Être affecté par des idées de choses à égalité avec les choses mêmes est le propre d'une sensibilité aux idées qui est la sensibilité intellectuelle même. Comme toutes les sensibilités [elle] doit sa formation à un certain type de trajectoire, à une certaine éducation, au sens le plus général du terme - celui où Flaubert parle d'éducation sentimental ${ }^{29}$.

Ces trajectoires relèvent sans doute de l'aléatoire, des rencontres des «lignes de vie » et de leurs métamorphoses. Mais la formation d'une sensibilité plus ou moins grande aux idées dépend aussi des inégalités et des mutilations que les sociétés divisées en classes engendrent et dont la reproduction constitue le cadre des trajets singuliers. La sensibilité intellectuelle correspond à un processus de séparation qui fait d'elle le privilège, ou l'handicap, social d'une couche particulière :

Le point de vue intellectuel sur le monde, qui se croit général, est frappé de sa particularité. C'est la plus commune des erreurs intellectuelles (...) que de penser la sensibilité intellectuelle unanimement partagée ${ }^{30}$. 
Cette «sensibilité " implique des attachements idiosyncrasiques, des «intérêts passionnés ", à l'égard des «idéalités " qui restent inégalement partagés: image renversée d'une activité humaine générale réellement libérée, l'amour et la maitrise des idées et des formes abstraites renvoient à la fois à l'émancipation et à l'aliénation, à l'égalité et à la séparation ${ }^{31}$. Le problème est donc celui de la généralisation de cette sensibilité par-delà les inégalités des rôles et des positions sociales :

Il s'agit que les abstractions idéelles gagnent en pouvoir d'affecter, et qu'elles deviennent capables de faire effet au-delà de cette minorité qu'on appelle usuellement les «intellectuels», ou bien, pour le dire autrement, que les intellectuels, compris non comme groupe social particulier mais comme disposition caractéristique, comme ingenium susceptible d'être affecté par des abstractions idéelles, deviennent de plus en plus nombreux ${ }^{32}$.

\section{« Imagination »}

44 Être affecté par des idéalités veut dire instaurer un rapport subjectif avec ce qui n'est pas donné ici et maintenant, ouvrir un espace subjectif-pratique par-delà les conditions existantes et viser donc une totalité virtuelle. C'est la sensibilité intellectuelle qui permet de penser et d'agir par-delà la situation présente et de s'orienter en fonction de quelque chose qui reste irréductible à l'actuel. F. Lordon qualifie d'« imagination » l'opérateur de cette sensibilité vis-à-vis des idées ${ }^{33}$.

Alexander Kluge et Oskar Negt ont eux aussi eu recours à la notion d'« imagination » pour qualifier la capacité des «forces productives intellectuelles » à dépasser les limites des rôles et des structures sociales et à se projeter dans l'invention de nouvelles possibilités :

La manière circonscrite et isolée dont le langage commun utilise le terme "imagination» est un produit de la bourgeoisie (...). Cette force productive et intellectuelle se voit classifiée en fonction de règles qui lui sont étrangères, celles $\mathrm{du}$ processus de valorisation capitaliste (...). Ce qui paraît difficilement domptable $\mathrm{du}$ point de vue de la valorisation - le résidu de souhaits inaccomplis, les idées et mouvements propres au cerveau qui se soustraient aux classifications bourgeoises est stigmatisé comme une pure fantaisie, une espèce de Tsigane ou de chômeur parmi les figures du travail intellectuel. Pourtant, cette imagination est en vérité un moyen de production particulier et nécessaire pour un travail que la valorisation capitaliste perd de vue : le changement des relations intrahumaines et envers la nature, ainsi que la réappropriation du travail mort que les hommes ont condensé dans l'histoire ${ }^{34}$.

Ainsi, le rapport de la pensée à une totalité non-donnée, sa capacité à dépasser toute condition objective, renvoie à ce lien que l'imagination instaure avec des sphères et des couches historiques qui ont été rendues inactuelles par un acte de refoulement. Le «besoin de la raison » vise donc tant une totalité à venir que des réalités perdues et effacées au cours de l'histoire de l'exploitation, et qui insistent en tant que figurations de l'imagination. C'est par cette insistance que les pouvoirs de la raison de transcender les données objectives sont rattachés aux métamorphoses et aux trajets archi-historiques des Gefühle. Par là, on voit que l'action de cette imagination intellectuelle est caractérisée par F. Lordon dans des termes peut-être excessivement restreints, qui relèvent d'ailleurs de l'objet même de son ouvrage, à savoir la dynamique mobilisatrice de l'« indignation »:

L'imagination intellectuelle étend la portée de la sympathie. Elle permet d'entrer dans les expériences, notamment les souffrances, d'une condition qui n'est pas la sienne ${ }^{35}$. 
47 besoin de la raison, mais le partage effectif des dispositions et des pratiques libératrices. L'indignation, bien que filtrée par les idéalités abstraites, ne saurait constituer par ses seules ressources subjectives une "volonté effective »; elle reste un affect régressif, car elle confirme et fige l'asymétrie entre ceux qui vivent directement une condition de souffrance et ceux qui en sont affectés par le biais du discours et de l'image.

Ainsi, comme le dit F. Lordon, « des gouffres d'incompréhension séparent » ceux qui sont les porteurs de la sensibilité intellectuelle et ceux qui sont écrasés par leur situation immédiate ${ }^{36}$. Or cette incompréhension ne peut être réduite par l'empathie face à la souffrance, qui reste un processus unidirectionnel, incapable de modifier le clivage immanent à la puissance des idéalités dans une société divisée en classes. C'est que cette incompréhension témoigne d'un double manque, qui frappe d'impuissance et de passivité tant ceux qui ont été constitués comme « intellectuels » que ceux qui ont été privés de l'accès au champ intensif des idéalités.

Car l'imagination intellectuelle n'est pas seulement un pouvoir qu'il s'agit d'universaliser par-delà ses porteurs sociologiques immédiats : elle est aussi une puissance qui s'étiole dans l'irréalité, du fait de rester le privilège de certaines couches sociales. Si la sensibilité aux idéalités est l'opérateur de la recherche d'une orientation, elle est en même temps, comme le suggèrent Kluge et Negt, le symptôme d'un manque qui relève de la division des puissances génériques de l'humanité. Or ce manque affecte tous les modes d'existence que produit cette division. Et c'est pourquoi un processus politique effectif ne se limite pas à généraliser des dispositions qui existent déjà dans le corps social, mais transforme leur nature au fur et à mesure qu'il les découple d'avec leur inscription dans des rôles sociaux déterminés. Alain Badiou l'a rappelé à juste titre :

L'activité politique réelle commence quand on est lié aux masses populaires de façon effective, et cela n'a guère de rapport avec le statut des intellectuels dans nos sociétés, lequel est un statut $d$ séparation et de privilège (...). La politique est une activité singulière, un trajet qui vous arrache à vos lieux et à vos déterminations sociales. Les intellectuels doivent aller aux portes des usines, dans les cités, dans les foyers. Les ouvriers et autres travailleurs ordinaires doivent aller dans des réunions, prendre de leur temps déjà très lourd pour étudier, discuter, décider, lire et diffuser des tracts... ${ }^{37}$

C'est dire que le vrai travail d'orientation, la vraie détermination d'une situation à partir du seul «besoin de la raison", ne devient effectif que lorsqu'il se confronte aux manques qui font trou dans l'ordre social et d'où peut se dégager l'aspiration vers une totalité, visée d'abord dans l'idéalité, mais qui ne peut rester dans l'idéalité sans devenir affabulation velléitaire et repli consolateur. Le problème n'est donc pas seulement celui de la généralisation de la puissance intellectuelle, mais aussi celui de ce qui manque à cette «sensibilité aux idées» pour devenir effective. Mais ce n'est pas aux « intellectuels » qu'il revient d'administrer ce qui manque à leur disposition singulière : ce n'est pas à eux qu'il revient d'opérer ce que Franco Fortini appelait la vérification de leurs propres pouvoirs et mandats ${ }^{38}$. Cette vérification ne peut se faire que dans et par ce que Badiou appelle « des alliances inattendues, des alliances improbables, des rencontres égalitaires » :

Il y a un prolétariat nomade venu des zones les plus dévastées (...). Cet énorme prolétariat nomade constitue une avant-garde virtuelle de la masse gigantesque des gens dont l'existence, dans le monde tel qu'il est, n'est pas prise en compte. Il y a 
aussi des intellectuels (...). Tout le problème est qu'ils se lient au prolétariat nomade, qu'ils aillent le voir, le consulter, parler avec lui ${ }^{39}$.

51 Par-delà l'empathie et la pédagogie unilatérale, ces rencontres réaliseraient ce que Fortini qualifiait de « pédagogie généralisée » : un processus où « tout le monde apprend à tout le monde et de tout le monde (...) ; un gigantesque dialogue ininterrompu $»^{40}-$ un dialogue dans lequel chaque partenaire est chargé de traiter et de travailler le manque de l'autre.

Ce n'est sans doute que par un pari, ou par un geste extrême de confiance, qu'il est possible de supposer l'existence des conditions de telles rencontres. Des conditions qui ne sont données que dans et par un besoin visant des choses «très anciennes mais oubliées", lesquelles ne sont visibles que dans l'espérance ${ }^{41}$. Peut-on considérer l'espérance comme l'un de ces Gefühle qui permettent de «tenir », d'articuler dans le présent les nœuds invisibles du passé et de l'avenir? C'est peut-être même la vertu fondamentale pour un tel rapport au temps, car en elle, et dans son corrélat qui est la patience, le passé et l'avenir sont reliés l'un à l'autre par une consistance commune, dont la métaphore est, depuis Hegel, la figure du travail, au sein duquel n'ont de cesse de se transformer l'un dans l'autre le maître et l'esclave, la pensée et le labeur, l'autosuffisance improductive et l'urgence du manque, la légèreté de l'esprit pur et le poids terrestre des gestes et des choses.

C'est à cette figure qu'Alain Badiou a recours pour caractériser la pratique systématique des rencontres émancipatrices. Ce n'est certainement pas anodin qu'il associe à cette figure du travail, qui relève de la dialectique du temps, celle du trajet, dont l'ouverture est décidée par le geste de l'orientation :

C'est un travail, qui est un travail pour tout le monde. C'est un travail de la pensée, c'est aussi un trajet: aller voir qui est cet autre dont on vous parle, qui il est vraiment. Il faut recueillir sa pensée, ses idées, sa vision des choses, et l'inscrire, lui, et vous en même temps, dans une vision stratégique du destin de l'humanité, qui essayera de faire que l'histoire de l'humanité oblique, change de direction et s'arrache au malheur opaque où en ce moment elle s'enfonce ${ }^{42}$.

\section{NOTES}

1. Alain Badiou, Un parcours grec, Paris, Lignes, 206, p. 7.

2. Ibid., p. 9

3. Immanuel Kant, Qu'est-ce que s'orienter dans la pensée ?(1786), introduit, traduit et annoté par Alexis Philonenko, Paris, Vrin, 2001, p. 87-88.

4. Ibid., p. 88.

5. Ibid., p. 94.

6. Alexander Kluge, Chronique des sentiments I, traduit sous la direction de Vincent Pauval, Paris, P.O.L., 2016, p. 991.

7. Ibid., p. 39.

8. Ibid.

9. A. Badiou, Un parcours grec, op. cit., p. 13.

10. Ibid., p. 12-13. 
11. Ibid., p. 13.

12. Ibid., p, 31.

13. Oskar Negt, «Travail et dignité humaine », in L'espace public oppositionnel, Paris, Payot, 2007, p. 182.

14. Alexander Kluge, Chronique des sentiments I, op. cit., p. 1032-1033.

15. Ibid., p. 1036.

16. Ibid., p. 1032.

17. Ibid.

18. Ibid., p. 1034-1035.

19. Ibid.

20. O. Negt, « Travail et dignité humaine », in L'espace public oppositionnel, op. cit., p. 182.

21. Ibid., p. 182-183.

22. Ibid., p. 179.

23. Alexander Kluge, Chronique des sentiments I, op. cit., p. 40.

24. Ibid., p. 68-69.

25. A. Badiou, Notre mal vient de plus loin. Penser les tueries du 13 novembre, Paris, Fayard, 2015, p. 61.

26. A. Badiou, Le siècle, Paris, Seuil, 2006 et De quoi Sarkozy est-il le nom ?, Paris, Lignes, 2007.

27. A. Badiou, Entretien platonicien (avec Maria Kakogianni), Paris, Lignes, 2015, p. 51.

28. Frédéric Lordon, Les affects de la politique, Paris, Seuil, 2016, p. 165-166.

29. Ibid., p. 166.

30. Ibid., p. 167.

31. Cf. Andrea Cavazzini, «Fragments sur le travail intellectuel », in Cahiers du GRM, IX, 2016, https://grm.revues.org/754.

32. F. Lordon, Les affects de la politique, op. cit., p. 169-170.

33. Ibid., p. 172.

34. A. Kluge-O. Negt, "Espace public et expérience ", in O. Negt, L'espace public oppositionnel, op. cit., p. 83.

35. F. Lordon, Les affects de la politique, op. cit., p. 174.

36. Ibid., p. 173.

37. A. Badiou, Entretien platonicien (avec Maria Kakogianni), op. cit., p. 51-52.

38. La « verifica dei poteri » est l'acte par lequel une assemblée examine la validité des mandats des élus. Elle a été introduite en France par la Révolution. Verifica dei poteri est le titre d'un recueil d'essai de Fortini publié en 1965, qui a exercé une influence considérable sur le marxisme critique et dans le débat sur les intellectuels et la politique

39. A. Badiou, Notre mal vient de plus loin. Penser les tueries du 13 novembre, op. cit., p. 61-62.

40. F. Fortini, «Dalla scuola alla piazza. Intervista con N. Agazzi », in Un dialogo ininterrotto. Interviste 1952-1994, Turin, Bollati-Boringhieri, 2003, p. 513.

41. Cf. A. Cavazzini, « "Des choses très anciennes mais oubliées". Notes sur Jean-Marie Straub et Danièle Huillet ", in Cahiers du GRM, VIII, 2015, https://grm.revues.org/739. Et surtout Charles Péguy, Note sur M. Bergson et Note conjointe sur M. Descartes, Liège, PULg, 2016.

42. A. . Badiou, Notre mal vient de plus loin. Penser les tueries du 13 novembre, op. cit., p. 63. 


\section{RÉSUMÉS}

Le texte développe le concept d'« orientation» proposé par Alain Badiou afin de cerner les impasses du moment politique actuel.

INDEX

Mots-clés : orientation, politique, intellectuels, Badiou Alain, Kant Immanuel, Kluge Alexander, Negt Oskar, Lordon Frédéric, Fortini Franco

Thèmes : philosophie, intellectuels, communisme

Index géographique : Europe

Index chronologique : XXe siècle

\section{AUTEUR}

\section{ANDREA CAVAZZINI}

Andrea Cavazzini est vice-président de l'Association-Groupe de Recherches Matérialistes. 